\title{
Land use Detection in Nusajaya using Higher-Order Modified Geodesic Active Contour Model
}

\author{
N. Alias ${ }^{1}$ \\ Center for Sustainable Nanomaterials \\ Ibnu Sina Institute for Scientific and Industrial Research \\ Universiti Teknologi Malaysia, UTM, Skudai, Johor, Malaysia
}

\author{
M. N. Mustaffa ${ }^{2}$, F. Mustapha ${ }^{3}$ \\ Department of Mathematical Sciences \\ Universiti Teknologi Malaysia, UTM \\ Skudai, Johor, Malaysia
}

\begin{abstract}
Urban development is a global phenomenon. In Johor, especially Nusajaya is one of the most rapidly developing cities. This is due to the increase of land demand and population growth. Moreover, land-use changes are considered to be one of the major components of current environmental monitoring strategies. In this context, image segmentation and mathematical model offers essential tools that can be used to analyze land use detection. The image segmentation process is known as the most important and difficult task in image analysis. Nonlinear fourthorder models had shown to have a good achievement in recovering smooth regions. Therefore, these motivate us to propose a fourth-order modified geodesic active contour (GAC) model. In the proposed model, a modified signed pressure force (SPF) function has been defined to segment the inhomogeneous satellite images. The simulations of the fourth-order modified GAC model through some numerical methods based on the higher-order finite difference method (FDM) have been illustrated. Matlab R2015a software in Windows 7 Ultimate on Intel (R) Core (TM) i5-3230M @ 2.60GHz CPU with 8 GB RAM has been considered as a computational platform for the simulation. Qualitative and quantitative differences between modified SPF functions and other SPF functions have been shown as a comparison. Hence land use detection is very useful for local governments and urban planners to enhance the future sustainable development plans of Nusajaya.
\end{abstract}

Keywords-Higher-order geodesic active contour (GAC); segmentation; land use; finite difference method; numerical methods

\section{INTRODUCTION}

Nowadays, land-use changes, increasing due to increases in human activities which then give a big impact on the global environmental changes [1]. Consequently, most ecosystems and the surface of the earth are significantly being modified by different kinds of human activities [1], [2] especially in forestry and urban development. Several techniques were formulated, applied and evaluated in order to detect the landuse changes [3], [4]. Recently, image segmentation has been an area of active research in the detection of medical and satellite images. The well-known nonlinear PDE-based tool for image segmentation is known as the geodesic active contour (GAC) model.

The GAC model is also known as an edge-based model [5]-[8]. The GAC model creates an edge indicator that enforces the development contour to the object boundaries [9]-[11]. However, the GAC model has difficulty converging at the right boundaries when it works on images with extreme noise or weak edges. The weaknesses of the GAC model were improved by introducing the shrinkage force. Author in [12] also adopted an error term in the generalized geodesic active contour (GGAC) model in order to detect the multi-connected region of images. This method, however, often suffers from serious problems with boundary leaks in images with weak object boundaries and the contour must be located close to the desired target, or the evolution curve can pitch to a local minimum and converge to the incorrect solution [13].

To resolve the leakage issue, Chan and Vese suggested a particular case with an active contour approach in which the original image intensity is homogeneity [13]-[15]. The aim of the method proposed by Chan and Vese is to find a contour in the segmented image. If the image intensity is inhomogeneity within or outside the image, the constants are not accurate. The Chan-Vese (CV) model subsequently does not generally segment images with intensity inhomogeneity. Therefore, the local information of the given image should be considered to segment the inhomogeneity images [12], [13], [16]. Some researchers have proposed an essential model to extract local information from inhomogeneity images. The proposed model is known as an active contour model based on local image fitting [12], [13], [16]. The other proposed model that considered the local information from inhomogeneity images is known as the local signed pressure force (SPF) function which utilized the advantages from both GAC and CV model [11], [17], [18]. This model, however, very sensitive to the initial contour placement and if the given images have adjacent objects, then it may cause failure in local segmentation.

Then [19] came up with the idea to resolve this problem by replacing the stopping function with a new global SPF function to effectively stop contours on weak edges. This modification is based on a homogeneous assumption that used the average of the inner and outer intensity of the curve. Therefore, the global SPF function is capable of controlling the evolutionary direction. Although the global SPF function is more efficient and can overcome the high cost of reinitialization, it is limited to segment blurred and complicated images. From this limitation, it motivates us to propose a new modified SPF function that uses the good in local and global SPF function. 
The GAC model was investigated as a helpful tool for image segmentation. In this paper, the fourth-order approximation has been implemented on the GAC model to enhance the accuracy of segmentation. The proposed fourthorder modified GAC model is based on the new modified SPF function that uses the edge and region information of the image. Therefore the aim of the proposed modified fourthorder GAC is to have achievement in recovering a smooth region and obtaining a better result in image segmentation. Furthermore, in the numerical part, the higher-order finite difference method (FDM) scheme has been utilized to develop the Penta-diagonal linear system of equation. Although this scheme is generally used in applied mathematics, only a few researchers have used this scheme to solve the image segmentation problem. Therefore, some numerical methods such as AGE, RBGS and JB methods will be used to solve the linear system of equation, which is a stable and easy method to implement.

In general, the purpose of this paper is to propose higherorder PDE-based segmentation to detect and monitor land-use changes in Nusajaya. Thus, the secondary objective is to improve the accuracy of land use segmentation. The rest of this paper is organized as follows. Section 2 is devoted to the descriptive study of the area. Related works on segmentation are presented in Section 3. Section 4 described the proposed segmentation model and some numerical methods. The proposed segmentation model was validated by several experiments and comparison results are presented in Section 5. A brief discussion based on the experiments is described in Section 6. Lastly is the conclusion of this paper as in Section 7.

\section{DESCRIPTION OF THE STUDY}

The study is located in the Nusajaya region of Johor as shown in Fig. 1. The new name of Nusajaya is known as Iskandar Puteri. This region has the fastest growing national economy. The Nusajaya region covers an area of $2217 \mathrm{~km} 2$ consisting of five local authorities. Furthermore, these five local authorities have five distinctive development points. Thus it guides its overall development. In 2010, Johor Baharu had $14.1 \mathrm{kha}$ of natural forest, extending over $38 \%$ of its land area. In 2017, it lost 572ha of natural forest.

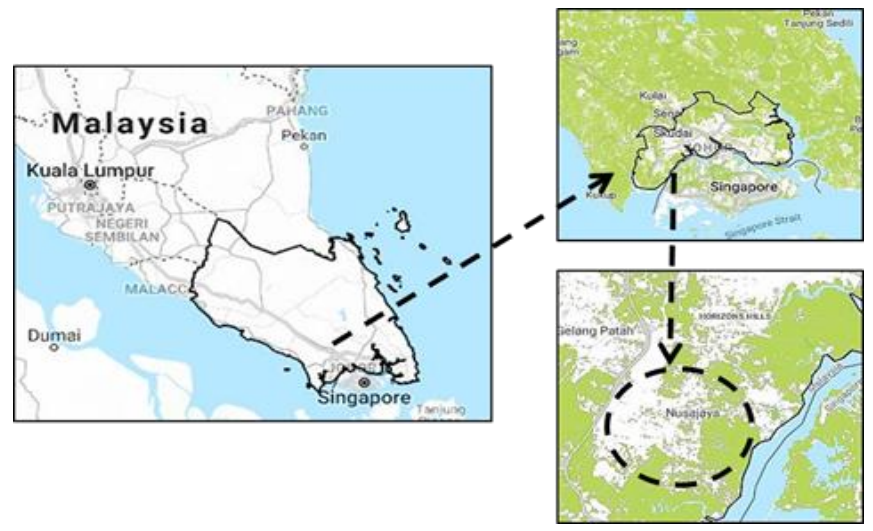

Fig. 1. Location of Study Area of Nusajaya, Johor Baharu, Malaysia. (https://www.globalforestwatch.org).

\section{RELATED WORK}

\section{A. The GAC Model}

The energy functional of the snake model introduced by Kass et al. (1988) can be simplified as

$E(\Gamma)=\int_{0}^{1} E_{\text {int }}(\Gamma(q))+E_{e x t}(\Gamma(q)) d q$

Note that, $\Gamma:[0,1] \rightarrow \mathfrak{R}^{2}$ is a parametrized curve, $E_{\text {int }}$ is the internal energy that controls the smoothness of the detected contours and $E_{e x t}$ is the external energy that is responsible for drawing contours towards the desired image features. Although (1) is successful due to its simplicity and efficiency, it is not intrinsic and has undesirable properties since it is dependent on the parameterization $q$, which is not related to the object boundary and it is also unable to change its topology. The active contour model is as follows [20]:

$$
\begin{aligned}
& \frac{\partial u}{\partial t}=g(I)|\nabla u| \operatorname{div}\left(\frac{\nabla u}{|\nabla u|}\right)+v g(I)|\nabla u| \\
& =g(I)(v+\kappa)|\nabla u|
\end{aligned}
$$

where $v$ is a positive real constant and $g(I)$ as stopping functions for instance:

$$
g=\frac{1}{1+|\nabla \hat{I}|^{p}}
$$

This stopping function $g(I)$ is responsible for controlling and stopping the iterative when it arrives at the desired image features. As a result, the edge-based model works well for images with clear edge information, but when it is used for detecting images with high noise and weak or blurred edges, it fails to reach the desired image features.

\section{B. CV Model}

Chan and Vese proposed a specific case with an active contour approach in which the original image intensity is uniform [13]-[15], [21]-[23]. The aim is to find a contour, $u$ in which the given image, $\boldsymbol{I}$ is segmented. The CV model is outlined in the following equation:

$$
\frac{\partial u}{\partial t}=\delta(u)\left[\mu \operatorname{div}\left(\frac{\nabla u}{|\nabla u|}\right)-\lambda_{1}\left(I(x)-c_{1}\right)^{2}+\lambda_{2}\left(I(x)-c_{2}\right)^{2}\right]
$$

where $\lambda_{1}, \lambda_{2}$ and $\mu$ are nonnegative constants. $c_{1}$ and $c_{2}$ represent the intensity averages for the inner or outer region.

The third term is the regularization length of the curve $u$. The following equations are defined for $c_{1}$ and $c_{2}$ :

$$
c_{1}(x)=\frac{\int_{\Omega} I(x) H(\phi(x)) \mathrm{dx}}{\int_{\Omega} H(\phi(x)) \mathrm{dx}}
$$


$c_{2}(x)=\frac{\int_{\Omega} I(x)(1-H(\phi(x))) \mathrm{dx}}{\int_{\Omega}(1-H(\phi(x))) \mathrm{dx}}$

As in (5) and (6), $c_{1}$ and $c_{2}$ are referred to both the internal and the external contour global properties of the image contents. If the intensity within or outside contour is not uniform, the constants are not accurate.

\section{The Local Region-based Model}

The GAC model is easily affected by noise and needs a long execution time, while the CV model is inefficient with intensity inhomogeneity and produces unsatisfactory segmentation results. According to [24], they have suggested a new local region-based SPF function in order to resolve this problem by replacing the stopping function, $g(I)$ and effectively stop the contours even on weak edges. The local region- based SPF function benefits from two smooth constants approximating the image intensities locally inside and outside the contour [24]. The SPF function is defined as

$$
\operatorname{spf}_{L C}=\frac{G_{\sigma}(x) * I(x)-\frac{f_{1}+f_{2}}{2}}{\max \left(\left|G_{\sigma}(x) * I(x)-\frac{f_{1}+f_{2}}{2}\right|\right)}, \quad x \in \Omega
$$

where $I(x)$ is the image, $\Omega$ is the image domain, $G_{\sigma}$ is the Gaussian kernel with standard deviation $\sigma$ and $*$ indicates the convolution operator. The denominator in (7) takes the maximum absolute value of the numerator. The SPF function is, therefore, will be derived within the range $[-1,1]$. Therefore $f_{1}$ and $f_{2}$ are defined as follows:

$$
\begin{aligned}
& f_{1}(x)=\frac{\int_{\Omega} G_{\sigma} * I(x) H_{\varepsilon}(\phi) \mathrm{d} \Omega}{\int_{\Omega} H_{\varepsilon}(\phi) \mathrm{d} \Omega} \\
& f_{2}(x)=\frac{\int_{\Omega} G_{\sigma} * I(x)\left(1-H_{\varepsilon}(\phi)\right) \mathrm{d} \Omega}{\int_{\Omega}\left(1-H_{\varepsilon}(\phi)\right) \mathrm{d} \Omega}
\end{aligned}
$$

The Heaviside function $H(\phi)$ in (8) was approximated by the smooth function $H_{\varepsilon}$ defined as

$$
H_{\varepsilon}=\frac{1}{2}\left[1+\frac{2}{\pi} \arctan \left(\frac{x}{\varepsilon}\right)\right]
$$

where $\varepsilon$ is a positive constant. $f_{1}$ and $f_{2}$ are the intensity averages for the inside and outside contour. Unfortunately, this local region-based SPF function can result in unsuccessful local segmentation when the images have adjacent objects. It is also easily affected by the initial contour placement [9], [11].

\section{The Global Region-based Model}

Consequently [19] proposed a new global SPF function to overcome the problem. Thus, by neglecting the term $\operatorname{div}\left(\frac{\nabla u}{|\nabla u|}\right)$, this model takes less time to converge and a fewer number of iterations to converge when compared with other models. The SPF function is defined as follows.

$$
\operatorname{spf}_{G L}=\frac{\left(c_{1} * c_{2}\right) * I(x)-\frac{c_{1}+c_{2}}{2}}{\max \left(\left|\left(c_{1} * c_{2}\right) * I(x)-\frac{c_{1}+c_{2}}{2}\right|\right)}, \quad x \in \Omega
$$

where $c_{1}$ and $c_{2}$ represent the intensity averages of regions inside $(u)$ or outside $(u)$.

$$
\begin{aligned}
& c_{1}(x)=\frac{\int_{\Omega} I(x) H(\phi) \mathrm{d} \Omega}{\int_{\Omega} H(\phi) \mathrm{d} \Omega} \\
& c_{2}(x)=\frac{\int_{\Omega} I(x)(1-H(\phi)) \mathrm{d} \Omega}{\int_{\Omega}(1-H(\phi)) \mathrm{d} \Omega}
\end{aligned}
$$

As a result, this global SPF function cannot detect all boundaries. This is due to (10) only using the global intensity information.

\section{PROPOSED MODEL}

\section{A. A Novel of Higher-Order Modified GAC Model}

This paper proposed a novel of higher-order modified GAC model that utilizes the advantages of the local regionbased model and global region-based model by integrating the local and global intensity information to develop a modified SPF function. The proposed modified SPF function is defined as:

$$
L C G L=\left(1-\omega_{1}\right)\left(s p f_{L C}-s p f_{G L}\right)+\omega_{1}\left(s p f_{G L}-s p f_{L C}\right)
$$

where $\omega_{1}=\operatorname{mean}(\operatorname{grad}(u))$ is the adaptive filter weight which helps to enhance the contrast of image. The main aim of modified GAC model is to detect the land-use changes in Nusajaya. The GAC approach allows the connection between the classical snake model (energy minimization) and the geometric active contour model (curve evolution theory). Therefore the GAC model allows stable boundary detection when the images have high gradients and gaps [20]. The main contribution of this paper is the new formulation of the fourthorder modified GAC model to enhance the capability for simulating and segmenting the land use on high-resolution satellite images of Nusajaya. Thus, the fourth-order modified GAC model is considered as follows:

$$
\begin{aligned}
\frac{\partial u}{\partial t} & =|\nabla u| \phi\left(\left(\frac{1}{(\Delta x)^{2}} \frac{\partial^{2} u}{\partial x^{2}}-\frac{(\Delta x)^{2}}{12} \frac{\partial^{4} u}{\partial x^{4}}\right)\right. \\
& \left.+\left(\frac{1}{(\Delta y)^{2}} \frac{\partial^{2} u}{\partial y^{2}}-\frac{(\Delta y)^{2}}{12} \frac{\partial^{4} u}{\partial y^{4}}\right)\right)+\left(1-\omega_{2}\right) v \operatorname{spf} f_{L C C L}|\nabla u|
\end{aligned}
$$


where $\phi=\frac{s p f_{L C G L}}{|\nabla u|}$ and $v$ is the balloon force that controls the contour expansion or shrinkage when the contour is inside or outside the object boundaries. Thus, $s p f_{L C G L}$ is the SPF function based on (12) and $\omega_{2}$ as the adaptive weight function adopting from [25] which then helps to allocate the role of the local and global SPF function. The following equation illustrates the central difference approximation from second-order to the fourth-order spatial accuracy for the first term of (13). This can result in approximation in (14).

$$
\begin{aligned}
\frac{u_{i, j}^{n+1}-u_{i, j}^{n}}{\Delta t} & =|\nabla u|_{i, j}^{n} \phi_{i, j}^{n}\left(\frac{-u_{i+2, j}^{n+1}+16 u_{i+1, j}^{n+1}-30 u_{i, j}^{n+1}+16 u_{i-1, j}^{n+1}-u_{i-2, j}^{n+1}}{12(\Delta x)^{4}}\right. \\
& \left.+\frac{-u_{i, j+2}^{n+1}+16 u_{i, j+1}^{n+1}-30 u_{i, j}^{n+1}+16 u_{i, j-1}^{n+1}-u_{i, j-2}^{n+1}}{12(\Delta y)^{4}}\right) \\
& +\mathrm{O}(\Delta t)+\mathrm{O}(\Delta x)^{4}+\mathrm{O}(\Delta y)^{4}
\end{aligned}
$$

Equation (14) has five-point center space with spatial truncation error of the order, $\mathrm{O}(\Delta x)^{4}$ and $\mathrm{O}(\Delta y)^{4}$. The model in (13) is discretized using a fourth-order central difference formula. Thus, this paper considers developing algorithms of a higher order, in particular, those whose accuracies are enhanced based on higher-order FDM scheme. Therefore, to realize this application, it is important to know that the discretization generates the fourth-order GAC model into a Penta-diagonal, instead of a tri-diagonal system of equations. The smaller truncation error of the fourth-order modified GAC model will give a more accurate representation of the numerical methods. The proposed fourth-order numerical methods are the JB, RBGS, and AGE methods.

\section{B. Fourth-Order Numerical Methods}

1) AGE method: In this paper, with reference to the work done by [26], the AGE4-PENTA method has been used to solve the fourth-order GAC model. This method is unconditionally stable. AGE is a suitable method because it has low computational complexity [27]. Therefore, the AGE method can be expressed as in the following algorithm:

a) Evolution in $x$-direction Calculate

$$
\begin{aligned}
& x^{\left(n+\frac{1}{2}\right)}=\left(G_{1}+r I\right)^{-1}\left\{\left(r I-G_{2}\right) x^{(n)}+f\right\} \\
& x^{(n+1)}=\left(G_{2}+r I\right)^{-1}\left\{\left(r I-G_{1}\right) x^{\left(n+\frac{1}{2}\right)}+f\right\}
\end{aligned}
$$

b) Evolution in y-direction Calculate

$$
\begin{aligned}
& y^{\left(n+\frac{1}{2}\right)}=\left(G_{1}+r I\right)^{-1}\left\{\left(r I-G_{2}\right) y^{(n)}+f\right\} \\
& y^{(n+1)}=\left(G_{2}+r I\right)^{-1}\left\{\left(r I-G_{1}\right) y^{\left(n+\frac{1}{2}\right)}+f\right\}
\end{aligned}
$$

2) RBGS method: Referring to the general iterative formula for the RBGS method in [28], the fourth-order RBGS can be expressed as in the equations below: a) Evolution in $x$-direction calculate

$$
\begin{aligned}
\text { for }^{i} & =3, \ldots, M-1 ; \\
x_{i}^{n+1} & =\frac{f_{i}+d_{x} x_{i-2}^{n}+a_{x} x_{i-1}^{n}+b_{x} x_{i+1}^{n}+e_{x} x_{i+2}^{n}}{\left(1+c_{x}\right)} \\
\text { for }^{i} & =2,4, \ldots, M \\
x_{i}^{n+1} & =\frac{f_{i}+d_{x} x_{i-2}^{n+1}+a_{x} x_{i-1}^{n+1}+b_{x} x_{i+1}^{n}+e_{x} x_{i+2}^{n}}{\left(1+c_{x}\right)}
\end{aligned}
$$

b) Evolution in y-direction calculate

$$
\begin{aligned}
\text { for } & j=3, \ldots, N-1 ; \\
y_{j}^{n+1} & =\frac{f_{j}+d_{y} y_{j-2}^{n}+a_{y} y_{j-1}^{n}+b_{y} y_{j+1}^{n}+e_{y} y_{j+2}^{n}}{\left(1+c_{y}\right)} \\
\text { for }^{i} & =2,4, \ldots, M \\
y_{j}^{n+1} & =\frac{f_{j}+d_{y} y_{j-2}^{n+1}+a_{y} y_{j-1}^{n+1}+b_{y} y_{j+1}^{n}+e_{y} y_{j+2}^{n}}{\left(1+c_{y}\right)}
\end{aligned}
$$

3) JB Method: Based on the general iterative formula of the JB method in [28], the fourth-order JB method can be represented by:

a) Evolution in $x$-direction calculate

for $i=2, \ldots, M-1$;

$x_{i}^{n+1}=\frac{f_{i}+d_{x} x_{i-2}^{n}+a_{x} x_{i-1}^{n}+b_{x} x_{i+1}^{n}+e_{x} x_{i+2}^{n}}{\left(1+c_{x}\right)}$

b) Evolution in y-direction calculate

for $j=2, \ldots, N-1$;

$y_{j}^{n+1}=\frac{f_{j}+d_{y} y_{j-2}^{n}+a_{y} y_{j-1}^{n}+b_{y} y_{j+1}^{n}+e_{y} y_{j+2}^{n}}{\left(1+c_{y}\right)}$

\section{Implementation}

Several steps are involved in this experiment. The experiment starts with reading and converting the highresolution satellite images of Nusajaya from signal to digital data. Then, the initial and boundary conditions of the proposed segmentation model are read and computed. The next step is employing and executing the numerical methods to solve the linear system of equation (LSE) of the fourth-order GAC model until convergence is achieved. Below is the algorithm for the proposed model.

\section{EXPERIMENTAL RESULTS}

The proposed model is applied to the high-resolution satellite image of Nusajaya adopted from Google Earth. The range of intensities of the image is represented from 0 to 255 , while the size in pixels (length $\times$ width) of the image is 200x200. The experiments developed by using Matlab R2015a software in Windows 7 Ultimate on Intel (R) Core (TM) i53230M @ 2.60GHz CPU with 8 GB RAM. In the proposed 
model, the following parameters are used: $\tau=1, \Delta x=\Delta y=1$, $v=-3, \sigma=1$ and $m=200 \times 200$.

\section{A. Comparison with Local Region-Based Model and Global Region-Based Model}

In order to test the modified SPF function, the proposed model in (13) will be compared with the local region-based model and global region-based model. A visualization and quantitative analysis based comparison between the segmentation results of four different years of high-resolution satellite images of Nusajaya is obtained. The ground truth images and segmented images are used to calculate the false positive (FP), true positive (TP), false negative $(\mathrm{FN})$, and true negative (TN) [11], [24].

Fig. 2 shows the comparison results of the global regionbased model, local region-based model and the proposed fourth-order modified GAC model for high-resolution satellite images with weak boundaries and noise. From top to bottom, the images in the first row are the satellite images of Nusajaya in the year 2006, and this image is quite weak with intensity inhomogeneity; the second row and the third row are the satellite images in the year 2009 and 2012, respectively, with intensity inhomogeneity weak boundaries; then the fourth row is the satellite images year 2015, and this image has severe intensity inhomogeneity. The ground truth and the final contour of the proposed model, global region-based model, and the local region-based model are plotted as red contours, blue contours, green contours, and yellow contours, respectively. Fig. 2 reveals that the segmentation results of the global region-based model cannot obtain the correct boundaries for all images since the images have intensity inhomogeneity. Then, the local region-based model is unsatisfactory since this model only uses the local intensity information to classify the images. By integrating the global and local intensity information, the proposed model successfully detects the object boundaries with high accuracy, sensitivity, and specificity.

Table I shows a quantitative analysis based on the computed segmentation results from Fig. 2. As shown in Table I, the proposed modified SPF function provides high accuracy and slightly fewer numbers of iterations and execution time as compared to the methods of [24] and [19]. From the results in Table I, the robustness of the proposed model has been proved to segment the satellite images of Nusajaya with intensity inhomogeneity and weak boundaries and thus better than the other two models.

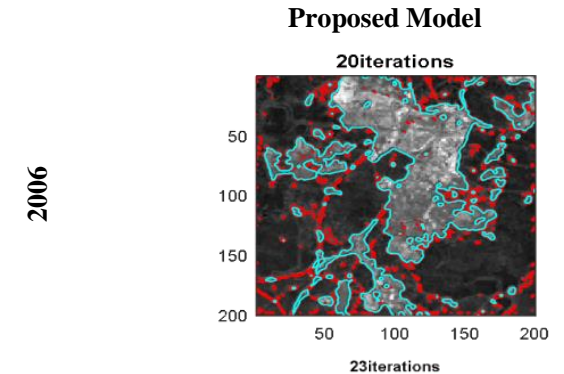

Global Region-Based Model
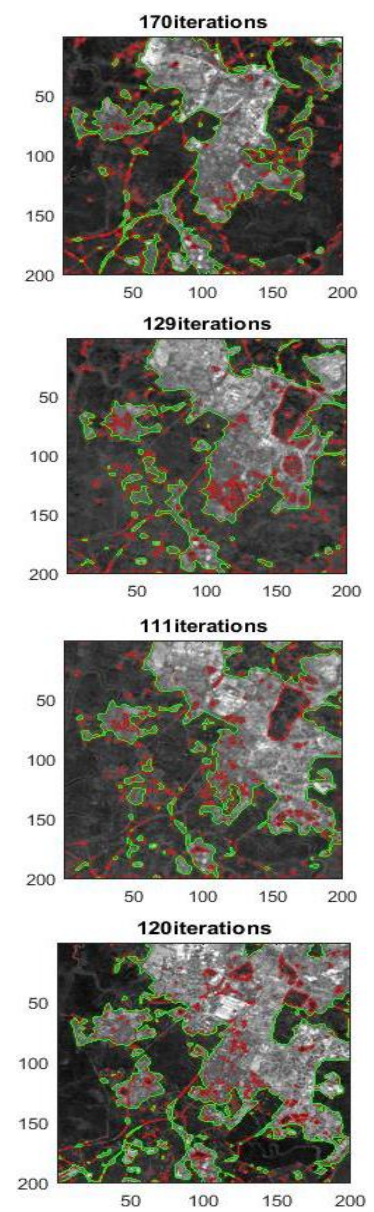

Local Region-Based Model
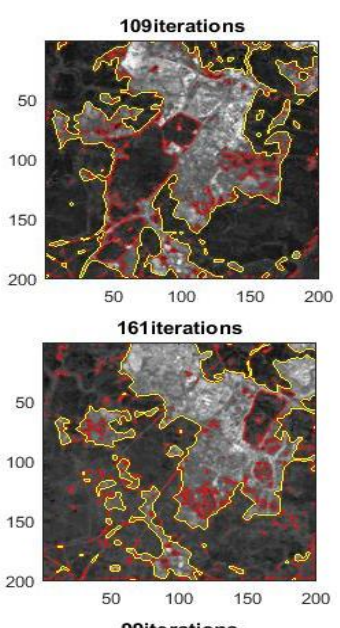

99iterations
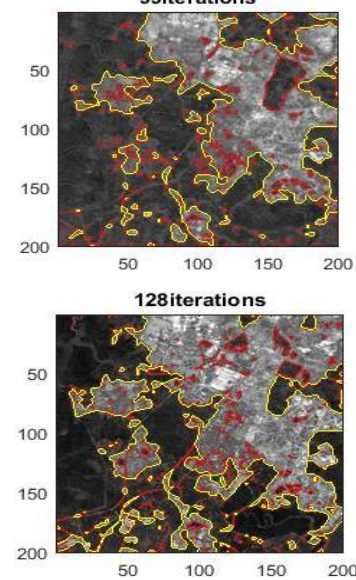

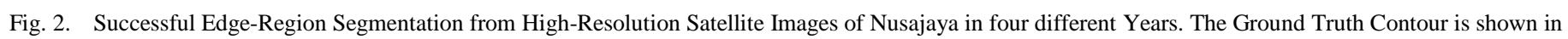

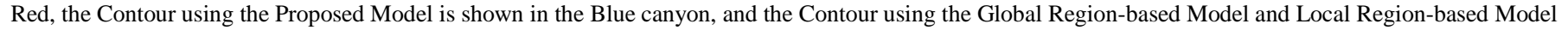
are Shown in Green and Yellow, Respectively. 
TABLE. I. QUANTITATIVE ANALYSIS BASED ON FIg. 2

\begin{tabular}{|c|c|c|c|c|c|c|}
\hline Model & Year & Accuracy & Specificity & Sensitivity & Execution Time & No. of Iterations \\
\hline \multirow{4}{*}{ Proposed Model } & 2006 & 0.9401 & 0.9131 & 0.9555 & 24.6014 & 20 \\
\hline & 2009 & 0.9269 & 0.8614 & 0.9642 & 29.0942 & 23 \\
\hline & 2012 & 0.9731 & 0.9053 & 0.9563 & 22.0897 & 18 \\
\hline & 2015 & 0.9355 & 0.9820 & 0.8943 & 10.2649 & 20 \\
\hline \multirow{4}{*}{ Global Region-Based Model } & 2006 & 0.9352 & 0.8880 & 0.9620 & 35.4278 & 172 \\
\hline & 2009 & 0.9083 & 0.9268 & 0.8978 & 27.1598 & 129 \\
\hline & 2012 & 0.9169 & 0.9418 & 0.9018 & 20.3581 & 111 \\
\hline & 2015 & 0.9209 & 0.9786 & 0.8698 & 25.7558 & 120 \\
\hline \multirow{4}{*}{ Local Region-Based Model } & 2006 & 0.8341 & 0.9771 & 0.7529 & 32.4950 & 109 \\
\hline & 2009 & 0.9066 & 0.9315 & 0.8924 & 60.6064 & 161 \\
\hline & 2012 & 0.9129 & 0.9451 & 0.8933 & 32.0270 & 99 \\
\hline & 2015 & 0.9209 & 0.9785 & 0.8687 & 48.8751 & 128 \\
\hline
\end{tabular}

Based on the quantitative analysis results, the proposed model has better accuracy performance than the other two models. This is because the proposed model can separate well the background and foreground of the image and able to converge at the correct boundaries. The execution time and iterations of the segmentation are presented in Table I. The proposed model consumes less time with less number of iterations to obtain satisfactory results. By contrast, from Table I, the local region-based model consumes the longest execution time with the most number of iteration among all the consideration models. So overall, the proposed fourthorder modified GAC model was superior with respect to the accuracy, number of iterations and less execution time.

\section{B. Comparison with a different value of $\sigma$}

An experiment has been conducted with the satellite images in order to validate the robustness of the proposed fourth-order modified GAC model and its segmentation accuracy with a different value of $\sigma$.
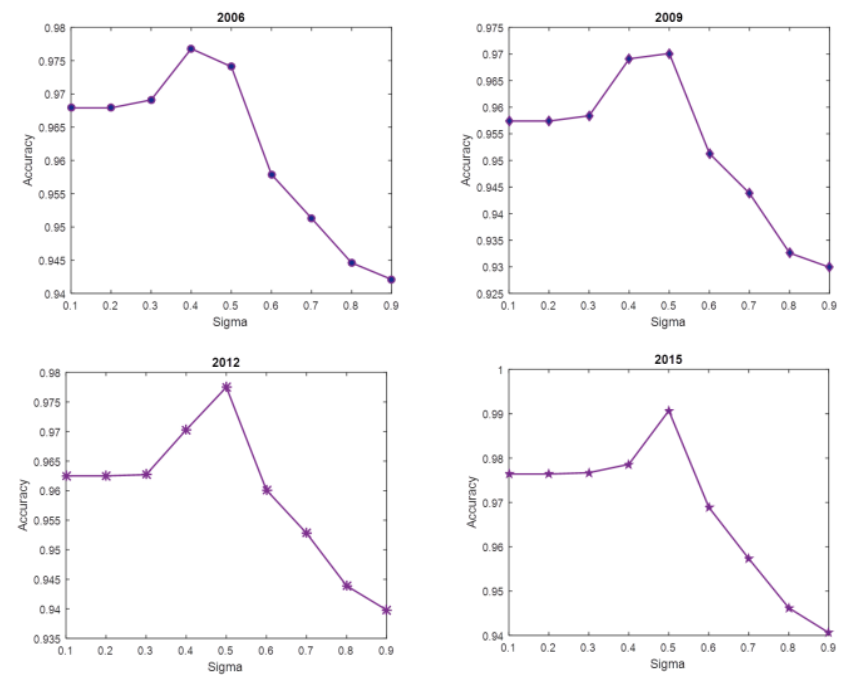

Fig. 3. Finding Optimum Accuracy with the difference Value of $\sigma$.
Fig. 3 shows the effect of the value of $\sigma$ on the segmentation results of the proposed fourth-order modified GAC model. Fig. 3 reveals that when the value $\sigma=0.4$, then the segmentation from satellite images year 2006 obtain high accuracy performance compared the other two tested values of $\sigma$. However, when setting the value of $\sigma=0.5$ then the segmentation results for satellite images in years 2009, 2012 and 2015 obtain high accuracy performance compared to the other two tested values of $\sigma$. Therefore, this clearly validates that the optimum accuracy of the proposed model depends on the images and value of $\sigma$.

\section{Comparison with different Numerical Method}

This subsection focuses on the implementation of some numerical methods on the proposed fourth-order modified GAC model. This experiment has been conducted using the following parameters: $\tau=1, \Delta x=\Delta y=1, v=-3$, tol $=1 e^{-3}$ and $m=200 \times 200$. In order to obtain a better segmentation with high accuracy, the value of $\sigma$ for satellite images in the year 2006 is set as $\sigma=0.4$ and the other three satellite images are set as $\sigma=0.5$. Table II shows the numerical results for high-resolution satellite images of Nusajaya. As depicted in Table II, the AGE method gives an excellent segmentation performance with less number of iterations, consume less execution time and less error compared to RBGS and JB methods. This clearly validates the superiority of the AGE method in the proposed segmentation model.

\section{DISCUSSION}

\section{A. Discussion on the Value of $\sigma$}

The value of $\sigma$ plays an important role in the proposed model to segment the high-resolution satellite images of Nusajaya. If the value of $\sigma$ is too small, the segmentation on land-use changes will not converge at the accurate boundaries. Otherwise, if the value of $\sigma$ is too high, the results will give less accurate segmentation. In application, this paper suggests that the value of $\sigma$ should be set in the range between 0.3 and 0.5 for most images. This can be seen in the graph plotted in Fig. 4 developed by using Matlab R2015a software. 
TABLE. II. NUMERICAL ANALYSIS RESULT

\begin{tabular}{|c|c|c|c|c|c|}
\hline 离 & Method & $\begin{array}{l}\text { Execution } \\
\text { Time }\end{array}$ & $\begin{array}{l}\text { No. of } \\
\text { Iterations }\end{array}$ & $\begin{array}{l}\text { Maximum } \\
\text { Error }\end{array}$ & RMSE \\
\hline \multirow{5}{*}{ ஜ્ণ } & AGE & 19.6139 & 3 & $5.2283 \mathrm{e}-04$ & $2.6141 \mathrm{e}-06$ \\
\hline & $\%$ & 74.75 & 96.51 & 46.16 & 74.38 \\
\hline & RBGS & 35.1314 & 30 & $7.9289 \mathrm{e}-04$ & $5.3551 \mathrm{e}-06$ \\
\hline & $\%$ & 54.77 & 65.12 & 18.36 & 47.52 \\
\hline & JB & 77.6728 & 86 & $9.7117 \mathrm{e}-04$ & $1.0205 \mathrm{e}-05$ \\
\hline \multirow{5}{*}{ ڤ્ণ } & AGE & 15.8908 & 3 & 7.9277e-04 & $3.9638 \mathrm{e}-06$ \\
\hline & $\%$ & 83.80 & 96.67 & 2.23 & 55.37 \\
\hline & RBGS & 45.4586 & 32 & $8.1261 \mathrm{e}-04$ & $5.7283 e-06$ \\
\hline & $\%$ & 52.45 & 64.44 & 4.79 & 35.51 \\
\hline & JB & 95.6130 & 90 & $7.7545 \mathrm{e}-04$ & $8.8819 \mathrm{e}-06$ \\
\hline \multirow{5}{*}{ 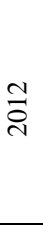 } & AGE & 13.8147 & 2 & $7.8261 \mathrm{e}-04$ & $3.9130 \mathrm{e}-06$ \\
\hline & $\%$ & 81.15 & 98.00 & 11.59 & 25.84 \\
\hline & RBGS & 28.5013 & 26 & $5.0318 \mathrm{e}-04$ & $5.2762 \mathrm{e}-06$ \\
\hline & $\%$ & 61.11 & 74.00 & 28.25 & 16.58 \\
\hline & JB & 73.2892 & 100 & $7.0132 \mathrm{e}-04$ & $6.3249 \mathrm{e}-06$ \\
\hline \multirow{5}{*}{$\frac{n}{2}$} & AGE & 16.5732 & 2 & $3.3148 \mathrm{e}-04$ & $1.6574 \mathrm{e}-06$ \\
\hline & $\%$ & 86.30 & 99.07 & 61.74 & 81.11 \\
\hline & RBGS & 31.4186 & 38 & $9.0516 \mathrm{e}-04$ & $6.8728 \mathrm{e}-06$ \\
\hline & $\%$ & 74.02 & 82.41 & 4.47 & 21.66 \\
\hline & JB & 120.9319 & 216 & $8.6642 \mathrm{e}-04$ & $8.7728 \mathrm{e}-06$ \\
\hline
\end{tabular}

\section{B. Discussion on Numerical Method Implementation}

With regard to the rate of convergence, the achievements of the AGE method can be seen clearly from the results in Table II in terms of execution times and number of iterations. For all images, the AGE method is $74.75 \%-86.30 \%$ better than JB method in terms of execution times. AGE method is much better than the RBGS and JB. Even though the RBGS and JB methods are derived from fourth-order approximation of the GAC model, but they are lacking in terms of accuracy due to the rounding off errors that have been accumulated from the start of execution until the end. Amongst all the tested methods, the AGE method still maintains its greater accuracy in all satellite images of Nusajaya. In general, the proposed higher-order PDE-based segmentation employing fourth-order numerical methods provides a class of computationally efficient, convergent and highly accurate solutions for land use segmentation of high-resolution satellite images.

\section{Discussion on Land-use Changes}

Fig. 4 displays the segmentation visualization of land use in Nusajaya. White color is as land use and black color as the land cover. Thus, this can be seen that each year, the land uses become increasing and less land cover which might cause a negative effect on the earth's balance. It can be predicted the land cover in the Nusajaya area might decrease by around $0.87 \%$ every year. In the year 2006, the land cover of the Nusajaya area is only $63.17 \%$ and the rest is land use. Additionally, every three years, the land cover has been decreased to $62.98 \%$ for the year $2009,60.01 \%$ for the year 2012 and $52.75 \%$ for the year 2015 .
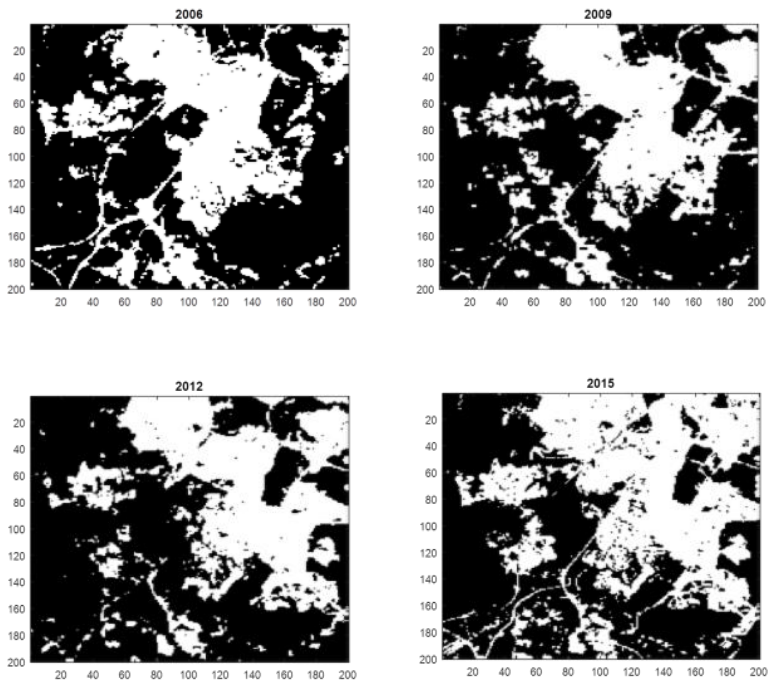

Fig. 4. Land use and Land Cover in Nusajaya in four different Years.

\section{CONCLUSION}

In this paper, there are two contributions. First is the development of modified SPF function which integrates the local and global intensity information to detect land-use changes in Nusajaya. The second contribution is the construction of fourth-order modified GAC model to enhance the accuracy of segmentation. The local intensity information makes the model works well for images with intensity inhomogeneity. Then, the global intensity information helps the model to converge faster. Furthermore, the proposed segmentation model has been implemented with an adaptive filter weight on the SPF function able to smoothly and successfully segment the high-resolution satellite images of Nusajaya. Therefore, the adaptive filter weight helps the proposed segmentation model to avoid expensive computational regularization. Experiments show that the proposed higher-order modified GAC model can accurately segment satellite images with intensity inhomogeneity and weak boundaries. In addition, the implementation of the AGE method of the proposed model is more superior to the other numerical methods. However the value of $\sigma$ needs to be well chosen depends on the image because the accuracy of segmentation may be affected. In the future, this work will be focused on reducing its computational complexity by implementing high-performance computing into the segmentation process.

\section{ACKNOWLEDGMENT}

This work was financially supported by the Universiti Teknologi Malaysia and Ministry of High Education (MOHE).

\section{REFERENCES}

[1] Giri, Remote sensing of land use and land cover: principles and applications. 2016.

[2] A. Alqurashi, L. K.-A. in R. Sensing, and undefined 2014, "Land use and land cover change detection in the Saudi Arabian desert cities of Makkah and Al-Taif using satellite data," 139.59.98.9.

[3] P. Srivastava, D. Han, M. Rico-Ramirez, ... M. B.-A. in S., and undefined 2012, "Selection of classification techniques for land use/land cover change investigation," Elsevier. 
[4] J. K. Thakur, P. K. Srivastava, S. K. Singh, and Z. Vekerdy, "Ecological monitoring of wetlands in semi-arid region of Konya closed Basin, Turkey,” Reg. Environ. Chang., vol. 12, no. 1, pp. 133-144, Mar. 2012.

[5] S. Yuan, P. Monkam, S. Li, H. Song, and F. Zhang, "Active contour model via local and global intensity information for image segmentation," Chinese Control Conf. CCC, pp. 5618-5623, 2017.

[6] M. A. Shafiq, Z. Wang, and G. Alregib, "Seismic interpretation of migrated data using edge-based geodesic active contours," 2015 IEEE Glob. Conf. Signal Inf. Process. Glob. 2015, pp. 596-600, 2016.

[7] Y. Luo et al., "Myocardial iron loading assessment by automatic left ventricle segmentation with morphological operations and geodesic active contour on $\mathrm{T} 2 *$ images," nature.com.

[8] J. Sun, N. Ray, and H. Zhang, "VFCCV snake: A novel active contour model combining edge and regional information," 2014 IEEE Int. Conf. Image Process. ICIP 2014, pp. 927-931, 2014.

[9] F. Akram, J. H. Kim, and K. N. Choi, "Active contour method with locally computed signed pressure force function: An application to brain MR image segmentation," Proc. - 2013 7th Int. Conf. Image Graph. ICIG 2013, pp. 154-159, 2013.

[10] F. Akram, J. H. Kim, H. U. Lim, and K. N. Choi, "Segmentation of intensity inhomogeneous brain MR images using active contours," Comput. Math. Methods Med., vol. 2014, 2014.

[11] F. Akram, J. H. Kim, C. G. Lee, and K. N. Choi, "Segmentation of regions of interest using active contours with SPF function," Comput. Math. Methods Med., vol. 2015, 2015.

[12] J. Yuan, J. Wang, and L. Liu, "Active contours driven by local intensity and local gradient fitting energies," Int. J. Pattern Recognit. Artif. Intell., vol. 28 , no. 3, 2014

[13] L. Zhang, X. Peng, G. Li, and H. Li, "A novel active contour model for image segmentation using local and global region-based information," Mach. Vis. Appl., vol. 28, no. 1-2, pp. 75-89, Feb. 2017.

[14] V. C. Korfiatis, P. A. Asvestas, and G. K. Matsopoulos, "Automatic local parameterization of the Chan Vese active contour model's force coefficients using edge information," J. Vis. Commun. Image Represent., vol. 29, pp. 71-78, 2015.

[15] S. Mukherjee and S. T. Acton, "Region-based segmentation in presence of intensity inhomogeneity using legendre polynomials," IEEE Signal Process. Lett., vol. 22, no. 3, pp. 298-302, 2015.

[16] J. Shi, J. Wu, A. Paul, L. Jiao, and M. Gong, "A partition-based active contour model incorporating local information for image segmentation," Sci. World J., vol. 2014, 2014.
[17] J. Yu, H. Guo, C. Li, J. Lu, and C. Jiang, "A waterline extraction method from remote sensing image based on quad-tree and multiple active contour model," Cehui Xuebao/Acta Geod. Cartogr. Sin., vol. 45, no. 9 , pp. 1104-1114, 2016.

[18] X. Li, D. Jiang, Y. Shi, and W. Li, "Segmentation of MR image using local and global region-based geodesic model," Biomed. Eng. Online, vol. 14, no. 1, pp. 1-16, 2015.

[19] Z. S. (2016) Reddy VL, "Active Contours With New Signed Pressure Force Function For Echocardiographic Image Segmentation," no. 4, pp. 3674-3678, 2016.

[20] Z. B. M. Zubaidin, "Alternating Group Explicit Method for Edge Detection on Brain and Breast Tumor Images.," Universiti Teknologi Malaysia, 2013.

[21] S. Liu and Y. Peng, "A local region-based ChanVese model for image segmentation,” Pattern Recognit., vol. 45, no. 7, pp. 2769-2779, 2012.

[22] P. Getreuer, "Chan - Vese Segmentation Simplified Mumford - Shah Model Level Set Functions," vol. 2, pp. 1-11, 2012.

[23] P. Ahmadi, S. Sadri, R. Amirfattahi, and N. Gheissari, "Automatic aerial image segmentation based on a modified Chan-Vese algorithm," 2012 5th Int. Congr. Image Signal Process. CISP 2012, no. Cisp, pp. 643-647, 2012.

[24] H. Zhang, J. Liu, Z. Zhu, and H. Li, "An automated and simple method for brain MR image extraction,” Biomed. Eng. Online, vol. 10, pp. 1-12, 2011.

[25] F. Dong, Z. Chen, and J. Wang, "A new level set method for inhomogeneous image segmentation," Image Vis. Comput., vol. 31, no. 10, pp. 809-822, 2013.

[26] E. S. Uzezi and G. Kparo, "Domain Decomposition of the Fourth-Order AGE Method on Heat Equation with MPI," Int. J. Comput. Appl., vol. 32, no. 10, pp. 25-38, 2011.

[27] N. Alias, H. F. S. Saipol, A. C. A. Ghani, and M. N. Mustaffa, "Parallel performance comparison of alternating group explicit method between parallel virtual machine and Matlab distributed computing for solving large sparse partial differential equations," Adv. Sci. Lett., vol. 20, no. 2, pp. 477-482, 2014.

[28] M. N. Mustaffa, N. Alias, and F. Mustapha, "Some numerical methods for solving geodesic active contour model on image segmentation process," Malaysian J. Fundam. Appl. Sci., vol. 13, no. 4-1, pp. 408$411,2017$. 